\title{
Nanotecnología y salud animal: alianzas, políticas públicas y aprendizajes en torno al desarrollo de una estrategia para prevención y tratamiento para la brucelosis ovina
}

\author{
Carrozza, Tomás Javier ${ }^{1,2}$; Susana Silvia Brieva ${ }^{1}$ \\ ${ }^{1}$ Departamento de Ciencias Sociales, Facultad de Ciencias Agrarias-Universidad Nacional de Mar del Plata, \\ Balcarce, Buenos Aires, Argentina; ${ }^{2}$ tomascarrozza@gmail.com
}

Carrozza, Tomás Javier; Susana Silvia Brieva (2020) Nanotecnología y salud animal: alianzas, políticas públicas y aprendizajes en torno al desarrollo de una estrategia para prevención y tratamiento para la brucelosis ovina. ${ }^{\omega}$ Rev. Fac. Agron. Vol 119 (1): 1-11. https://doi.org/10.24215/16699513e041

A partir del uso del enfoque socio-técnico, este trabajo busca reconstruir el proceso de I+D para el tratamiento de la brucelosis ovina mediante nanotecnologías, a fin de contribuir al debate sobre el papel de las tecnologías emergentes en el desarrollo de estrategias de salud animal. Las Nanotecnologías se han convertido en un tema central en la agenda de políticas en el cual, las problemáticas relacionadas al sistema agroalimentario han recibido un gran número de recursos. Entre los resultados se destaca que este desarrollo generó nuevas capacidades y aprendizajes. Sin embargo, se plantean conflictos acerca de la utilidad social del conocimiento generado.

Palabras clave: agronanotecnologías, salud animal, sistema agroalimentario argentino, enfoque socio técnico

Carrozza, Tomás Javier; Susana Silvia Brieva (2020) Nanotechnology and animal health: learning about the development of a strategy for prevention and treatment for ovine brucellosis. Rev. Fac. Agron. Vol 119 (1): 111. https://doi.org/10.24215/16699513e041

From a socio-technical approach, the aim of this paper is to reconstruct the R\&D process for the treatment of ovine brucellosis through nanotechnologies. This research wants to contribute to the debate on the role of emerging technologies in the development of animal health strategies. Nanotechnologies have become a central topic in the policy agenda in which the problems related to the agro-food system have received a large number of resources. As a result, we highlight that this development generate new capacities and learnings. However, conflicts arise about the social utility of the generated knowledge.

Keywords: agronanotechnologies, animal health, argentine agrifood system, sociotechnical approach

https://revistas.unlp.edu.ar/revagro

Recibido: 31/07/2019

Aceptado: 30/09/2019

Disponible on line: $01 / 07 / 2020$

ISSN 0041-8676 - ISSN (on line) 1669-9513, Facultad de Ciencias Agrarias y Forestales, UNLP, Argentina 


\section{INTRODUCCIÓN ${ }^{1}$}

Desde hace varias décadas la producción ovina en Argentina se encuentra en disminución ${ }^{2}$, no obstante, en algunas regiones -como la Patagonia ${ }^{3}$ - aún posee un alto peso relativo dentro de los sistemas agrícolaganaderos. Como consecuencia, siguen existiendo demandas hacia el sistema científico-tecnológico en la búsqueda de soluciones a las diferentes problemáticas que plantea esta producción.

Entre estas, la brucelosis ovina es una patología que aún sigue prevaleciendo en gran parte de los establecimientos. Para su solución y combate desde el año 2010, investigadores del Instituto Nacional de Tecnología Agropecuaria (INTA) trabajan en un proyecto basado en el desarrollo de soluciones para la problemática de la Brucelosis Ovina mediante la aplicación de nanotecnologías (N\&N).

En este trabajo se reconstruye analíticamente el proceso de I+D para el diagnóstico y tratamiento mediante una vacuna- de la brucelosis ovina. El análisis de la trayectoria contribuye además al debate sobre el papel de las nanotecnologías en el desarrollo de estrategias de salud animal en Argentina.

El trabajo se organiza de la siguiente manera. En primer lugar, se realiza un repaso por aquellos estudios que relacionan los aportes de las nanotecnologías al sector agroalimentario. Posteriormente se introduce la perspectiva teórica utilizada en el trabajo. A continuación, se analizan y comparan las metodologías de prevención, diagnóstico y combate para Brucelosis Ovina en nuestro país. Así, se reconstruyen en forma estilizada las alianzas socio-técnicas en torno a cada uno de los métodos $\mathrm{y}$, por último, se presenta una síntesis del análisis realizado.

\section{Nanotecnologías para el sector agroalimentario: un breve repaso por su bibliografía}

Desde principios de la década de 2000 los investigadores consideran que las N\&N pueden llegar a tener gran importancia en la producción de alimentos, abarcando aspectos tales como packaging, nanosensores de calidad alimentaria, sistemas de potabilización de agua, detectores de agroquímicos, sistema de salud veterinaria, entre otros (FAO/WHO, 2010; Noormans, 2010; Duncan, 2011, entre otros). Sin embargo, autores como (Sozer \& Kokini, 2009) afirman que las aplicaciones y posibles impactos de las $N \& N$ en el sector agroalimentario y agroindustrial son relativamente recientes en comparación con otros sectores como la microelectrónica o el drug delivery, En los últimos años, la producción bibliográfica alrededor de esta temática comenzó a aumentar su relevancia generando un vasto conjunto de

\footnotetext{
1 Una versión previa de este trabajo fue presentada en Carrozza \& Brieva (2015).

2 "En las últimas décadas, el cambio en los sistemas de producción, el avance de la frontera agrícola, el reemplazo del ganado ovino por bovino, la desertificación por sobre pastoreo en la Patagonia, y una falta de interés por adecuar la actividad son aspectos que generaron una fuerte retracción del stock ovino" Agüero et al. (2010).

${ }^{3}$ La Patagonia Argentina es una región conformada por las provincias de Neuquén, La Pampa, Río Negro, Chubut, Santa Cruz y Tierra del Fuego e Islas del Atlántico Sur.
}

publicaciones al respecto. Esto se dio particularmente en el ámbito internacional, entre las publicaciones realizadas ${ }^{4}$, encontramos:

Trabajos como el de Ali et al. (2014), coinciden en afirmar la relevancia de las $\mathrm{N} \& \mathrm{~N}$ en un conjunto de aplicaciones agroalimentarias similares a las nombradas anteriormente, pero construyen una relación directa con lo ocurrido en muchos países respecto de la relación con los OGM y las cuestiones asociadas al riesgo y la percepción pública.

De forma similar, Bernardes et al. (2014) realizan un relevamiento respecto del papel de las $N \& N$ en cuestiones tales cómo la nanoencapsulación de compuestos activos, los nanosensores especializados en patógenos y las aplicaciones del packaging en relación a la toxicología. Los mismos concluyen, que el papel del usuario es central para la aceptación de las $\mathrm{N} \& \mathrm{~N}$, como así también la necesidad de profundizar de forma similar al anterior- en los análisis de los riesgos.

En la misma línea, Bouwmeester et al. (2014) realizan un relevamiento de productos alimenticios que contienen nanoelementos, llegando a encontrar un total de 140 , los que varían ampliamente entre sí, destacándose el uso en preparados nutritivos y vitamínicos. A este trabajo se suman relevamientos similares como el de Chellaram et al. (2014), Dasgupta et al. (2015). Ditta el al. (2015), que trabajan en un relevamiento pero haciendo hincapié en las posibilidades de las $\mathrm{N} \& \mathrm{~N}$ en generar sistemas de producción sustentables.

A los trabajos previos, se suma lo realizado por Frewer et al. (2014), que realizan un relevamiento de la bibliografía existente en torno a las percepciones y las actitudes del público respecto del uso de nanotecnologías en alimentos. Si bien destacan los avances, surgen un conjunto de preguntas derivadas tanto de las consecuencias propias del uso de las N\&N en sí mismo, como así también de los aprendizajes derivados del uso de OGM y la necesidad de construir procesos de legitimación. En la misma línea, Handford et al. (2014) trabajan en las cuestiones asociadas a las oportunidades, beneficios y riesgos y concluyen que existe una "brecha" de conocimiento entre las cuestiones de I+D y los avances en las cuestiones asociadas al riesgo que son necesarias para construir regulaciones que permitan generar una implementación "plena" en la industria.

En esta línea, se suman a lo anterior los relevamientos de Kumari \& Yadav (2013), Myhr \& Myskja (2013), Agrawal \& Ratore (2014), Mihindukulasuriya \& Tim (2014), Mishra et al. (2014), Parisi et al. (2014), Prasad et al. (2014), Rossi et al. (2014), Samantarai \& Achakzai (2014), Vijayalakshmi et al. (2014); Warriner et al. (2014) y Mukhopadhyay (2015). Los mismos, aunque con matices buscan abarcar los esfuerzos de

\footnotetext{
${ }^{4}$ Gran parte de estos trabajos se publicaron en Trends in Food Science \& Technology 40 (2): 125-242 (December 2014). Special Issue: Nanotechnology in Foods: Science behind and future perspectives. Edited by Finglas, P.M., R.Y. Yada \& F. Toldrá.
} 
I+D realizados en $\mathrm{N} \& \mathrm{~N}$ para los diferentes aspectos del sistema agroalimentario y agroindustrial.

Dentro del grupo de trabajos que realizaron tareas de relevamiento se destaca lo realizado por Resch \& Farina (2015), los que trabajan en un mapa del conocimiento de las $\mathrm{N} \& \mathrm{~N}$ en el sector agroalimentario. Mediante la utilización de software, los autores realizan un relevamiento en el periodo 2001-2013 en cual resaltan cuatro tendencias: 1. Uso de biosensores, 2. Utilización de packaging "activo", 3. Encapsulamiento, 4. Riesgos, beneficios y marcos regulatorios.

Finalmente, dentro de los trabajos dedicados a analizar y relevar esta relación, Cortes-Lobos (2013) -en el marco de su investigación doctoral- releva lo ocurrido con la investigación en $\mathrm{N} \& \mathrm{~N}$ en el sistema sectorial de innovación agroalimentario en Estados Unidos. Destaca, en primer lugar, el aumento de investigaciones cuyo objetivo es una mejora en la sostenibilidad de la producción de alimentos. Paralelamente, al analizar los actores involucrados identifica una tensión entre los investigadores y agencias gubernamentales que impulsan este tipo de investigaciones y las asociaciones que ponen en duda estos avances como consecuencia de la falta de análisis de los riesgos.

Sin embargo, para esta área, aun no hay un consenso respecto del rol que pueden tener las N\&N. Mientras que algunos autores consideran que se asiste a la emergencia de un "nano-paradigma en las corporaciones alimentarias" (Scrinis \& Lyons, 2007), otros autores aun se cuestionan si más allá de las promesas en los cambios de la producción de alimentos nos encontramos frente a una moda más que frente a un verdadero cambio tecnológico (Busch, 2008).

Los trabajos a nivel regional y nacional son escasos. Autores como Foladori \& Invernizzi (2007), Delgado (2009) y García et al. (2010) focalizan sus estudios mayormente en los posibles impactos que van a tener las $\mathrm{N} \& \mathrm{~N}$ en el sector, destacando las grandes inversiones realizadas desde el sector privado en la búsqueda de "nanosoluciones" para alimentos procesados y mejoras en el manejo de cultivos.

En este marco, donde las Nanociencias y Nanotecnologías pueden generar grandes cambios en el sistema agroalimentario y agroindustrial, sistema en el cual nuestro país posee ventajas comparativas y competitivas, resulta oportuno analizar los procesos de I+D+i y la construcción social de las agendas de Ciencia y Tecnología.

\section{DESARROLLO}

La perspectiva analítica de las $\mathbf{N} \& N$ en el marco de los estudios sociales de la ciencia y tecnología

En el marco de los Estudios Sociales de la Ciencia y la Tecnología (ESCyT), desde una concepción constructivista, socio-técnica e interactiva de los procesos, en el análisis de los métodos de detección para la Brucelosis Ovina, se integran y complementan conceptualizaciones provenientes del enfoque sociotécnico y de política, abordajes potencialmente complementarios, pero aun escasamente interconectados o interrelacionados.
La perspectiva constructivista permite analizar el proceso de producción de conocimiento y desentrañar la compleja red de alianzas, estrategias y actores sociales involucrados en la construcción de conocimiento científico-tecnológico (Licha, 1995).

En el proceso de detección de la Brucelosis en los establecimientos ovinos convergen y entrelazan un conjunto de elementos y relaciones heterogéneas y complejas, como instituciones científicas y tecnológicas, ovejas, médicos veterinarios, investigadores, sangre, brucella ovis, vacunas, extensionistas, equipamiento, entre otras. En el análisis se recurre entonces, a conceptos pertenecientes al enfoque socio-técnico que posibilitan la reconstrucción analítica de las complejas relaciones y cambios entre usuarios y herramientas, actores y producciones, instituciones y sistemas tecnoproductivos asociadas, en este caso al funcionamiento del campo de conocimiento nanotecnológico.

El punto de partida para el análisis socio-técnico constructivista es la identificación de los grupos sociales relevantes (GSR), concepto que remite a instituciones, organizaciones, grupos de individuos que comparten un conjunto de significados y relaciones problema - solución, en este caso referidos al sistema agronanotecnológico. Los distintos GSR definen si las soluciones funcionan o no, de acuerdo a si cumplen o no con sus objetivos o propósitos. Bijker (1995) sostiene que el "funcionamiento" es una contingencia que se construye social, tecnológica, política y culturalmente. Así, el "funcionamiento" o "nofuncionamiento" es una relación y es resultado de un proceso de construcción socio-técnica en el que intervienen elementos heterogéneos: sistemas, conocimientos, regulaciones, materiales, financiamiento, prestaciones, etc. Hace referencia a una interacción entre humanos y no-humanos: usuarios y artefactos, diseñadores y prototipos, planificadores y sistemas, evaluadores y tecnologías. No se trata de una condición estable. Un proceso de construcción de funcionamiento / no funcionamiento es una secuencia: supone complejos procesos sucesivos de adecuación / inadecuación de soluciones tecnológicas a concretas y particulares articulaciones socio-técnicas, históricamente situadas. A su vez, la continuidad o discontinuidad de la condición de funcionamiento se sustenta en la articulación de alianzas socio-técnicas estables. Una alianza socio-técnica es, entonces, una coalición de elementos heterogéneos implicados en el proceso de funcionamiento - no funcionamiento de un artefacto o una tecnología (Thomas, 2009), en este caso referidos $u$ asociados al campo nanotecnológico argentino bajo estudio, y su contribución a la prevención de enfermedades de alta incidencia en la población y mejora al sistema de salud nacional.

En esta perspectiva, para describir y explicar en términos de proceso las relaciones socio-técnicas que se establecen y dar cuenta de la multiplicidad de dimensiones que intervienen en los procesos de construcción de un textil repelente basado en nanotecnologías, en el análisis se incluye la noción de trayectoria socio-técnica, que refiere al proceso de coconstrucción de productos, procesos productivos y organizaciones, instituciones, relaciones usuarioproductor, relaciones problema-solución, procesos de construcción de "funcionamiento" y "utilidad" de una 
tecnología, racionalidades, políticas y estrategias de un actor (institución de I+D, universidad, etc.), o, asimismo, de un marco tecnológico (Bijker, 2005) determinado. Este concepto -de naturaleza eminentemente diacrónica- permite ordenar relaciones causales entre elementos heterogéneos en secuencias temporales, tomando como punto de partida un elemento sociotécnico en particular (por ejemplo, una tecnología artefacto, proceso, organización determinada-, una empresa, un grupo de I+D). Las dinámicas sociotécnicas en cambio, son más abarcativas que las trayectorias: toda trayectoria socio-técnica se desenvuelve en el seno de una o diversas dinámicas socio-técnicas y resulta incomprensible fuera de ellas.

El uso de herramientas del análisis socio-técnico se complementa con conceptualizaciones proveniente de la economía de la innovación. Los teóricos de esta corriente distinguen, entre otros, los siguientes procesos de aprendizaje: i) aprender haciendo (learning by doing) introducido por Arrow (1962) para referirse a la posibilidad de obtener aumentos de productividad sin cambios mayores, a través del perfeccionamiento de las capacidades operativas de una determinada instalación productiva; ii) aprender por el uso (learnig by using) empleado por Rosenberg (1982) para señalar la acumulación progresiva de habilidades y experiencias que se adquieren por utilizar productos y/o procesos, que se hace cada vez más eficiente por el uso de los mismos; iii) aprender por interacción (learning by interacting) propuesto por Lundvall $(1985,1988,1992)$ para señalar que existe una relación muy estrecha entre los procesos de aprender haciendo y aprender usando, dado que ocurren en un espacio determinado y la interacción mutua se va enriqueciendo con el tiempo. Finalmente, para comprender el funcionamiento de las alianzas es necesario utilizar el concepto de poder, el cual es relacional, y tal como define Giddens (1979) es una capacidad de los actores de asegurar resultados cuando los mismos dependen de la agencia de otros. El poder es ejercido, antes que poseído, y se encuentra presente en las relaciones e interacciones.

A través de una estrategia metodológica de tipo cuanticualitativo de carácter diacrónica-sincrónica, en la investigación se integra la búsqueda, análisis y sistematización de la información secundaria disponible en instituciones públicas y privadas, tales como el Consejo Nacional de Investigación Científica y Tecnológica (CONICET), Fundación Argentina de Nanotecnología (FAN), Ministerio de Ciencia, Tecnología e Innovación Productiva (MINCyT), Instituto Nacional de Tecnología Agropecuaria (INTA), el Servicio Nacional de Sanidad y Calidad Agroalimentaria (SENASA) entre otras, y con la realización de entrevistas en profundidad a distintos actores participantes en el uso y desarrollo de las diferentes metodologías para la detección de la Brucelosis Ovina.

\section{La brucelosis y su diagnóstico: trayectoria de un problema ¿Público? ${ }^{5}$}

\footnotetext{
${ }^{5}$ Si bien la problemática de la Brucelosis Ovina conforma parte de las agendas de políticas de diferentes organismos de I+D estatales, no necesariamente la misma puede ser un problema de orden público. Según Gusfield (2014 [1981]), se debe distinguir entre problema privado y problema público, ya que
}

La brucelosis es una de las principales patologías asociadas a la producción ovina en Argentina. Esta enfermedad, de tipo infecciosa, es causada por Brucella Ovis y se distribuye a lo largo del país. La misma, considerada como el principal motivo en la generación de problemáticas reproductivas de ovinos, ha mostrado en algunos casos hasta un $20 \%$ de prevalencia en los establecimientos (López et al., 2010).

Al margen de la dispersión de las mediciones y la inexistencia de un Programa Nacional asociado al combate y erradicación de la patología, diferentes actores estiman que la prevalencia de esta enfermedad puede llegar hasta el $50 \%$ de individuos infectados. Procedente del genero Brucella, afecta diferentes funciones del sistema reproductivo de machos $y$ hembras y encuentra reportes en nuestro país desde mediados de los años `60 (Paolicchi, 2005; López 2010).

A nivel internacional, este agente infeccioso se encuentra en todas las zonas de producción de ovinos y de forma similar a Argentina, se le atribuye gran importancia a la hora de hacer referencia a las problemáticas reproductivas de los establecimientos (Querol Sanchis, 2011).

Tanto productores como instituciones estatales principalmente SENASA- recomiendan para su diagnóstico dos métodos: uno tradicional asociado al tacto de los animales, y uno de "tipo inmunológico" basado en el análisis de muestras de sangre. Recientemente, un conjunto de investigadores del INTA han comenzado a trabajar en el diseño de un equipo de diagnóstico basados en el uso de $N \& N$. (Robles, 2008).

Los dos primeros métodos, de amplia trayectoria en el país, se encuentran en coexistencia. La prevalencia de uno $u$ otro método, al momento de detectar la brucelosis, se basa en un conjunto de factores tales como: la ubicación geográfica, la especificidad en los resultados, las demandas de integrantes de institutos de I+D, entre otros.

En cambio, el método nano, se encuentra en etapa de desarrollo y pruebas de prototipo a campo y es presentado por los técnicos como una propuesta "superadora", ya que en su opinión a partir del uso de las N\&N se avanza en un método de alta especificidad y fácil utilización por parte del usuario final.

A continuación, se describen en forma estilizada las características socio-técnicas de los métodos de diagnóstico mencionados (tabla 1).

Los GSR atribuyen a los tres métodos de diagnóstico diferente funcionamiento y significado de acuerdo a sus características de: confiabilidad, dominancia, rapidez (en la obtención de resultados) y practicidad, que pueden agruparse a los fines de este estudio bajo el criterio de "funcionalidad de la técnica". Los GSR consideran estos atributos en forma conjunta, ya que los mismos son interdependientes.

cuando se enfatiza el aspecto público se resalta la importancia del proceso a través del cual se define el problema, el cual se lleva a cabo en una arena pública. 
Revista de la Facultad de Agronomía, La Plata (2020) Vol 119 (1): 1-11

Tabla 1. Características socio-técnicas de los métodos de diagnóstico. Fuente. Elaboración propia.

\begin{tabular}{|c|c|c|c|}
\hline Método diagnostico & Tacto & Inmunológico & Nano \\
\hline Confiabilidad & Baja & Alta & Alta \\
\hline Funcionalidad de la & Alta & Baja & - \\
\hline técnica & Alta & Baja & Alta \\
\hline Practicidad & Alta & Baja & Muy alta \\
\hline Régimen de Conocimientos & $\begin{array}{l}\text { Genéricos- } \\
\text { disciplinares }\end{array}$ & Disciplinar & Transdiciscplinares \\
\hline Tipo de conocimiento & $\begin{array}{c}\text { Tácito } \\
\text { Aprender- } \\
\text { Haciendo } \\
\text { Conocimiento } \\
\text { experto }\end{array}$ & Conocimiento experto & $\begin{array}{l}\text { Conocimiento experto } \\
\text { Aprender-Haciendo } \\
\text { Leaning by interacting }\end{array}$ \\
\hline Capacidades de I+D & Baja & Medias & Alta \\
\hline Articulación institucional & Escasa & Media & Alta \\
\hline $\begin{array}{l}\text { Asociación al mainstream científico- } \\
\text { tecnológico }\end{array}$ & Escasa & Media & Alta \\
\hline Relaciones usu ario-productor & Alta & Media-baja & Alta \\
\hline $\begin{array}{l}\text { Estrategia de resolución de problema } \\
\text { asociada }\end{array}$ & $\begin{array}{l}\text { Eliminación- } \\
\text { perdida de } \\
\text { ganado }\end{array}$ & $\begin{array}{l}\text { Eliminación perdida } \\
\text { de ganado }\end{array}$ & Vacuna nano vehicular \\
\hline GSR Involucrados & $\begin{array}{c}\text { Productor } \\
\text { Médicos } \\
\text { veterinarios } \\
\text { Instituciones de } \\
\text { I+D }\end{array}$ & $\begin{array}{c}\text { Productor } \\
\text { Laboratorio } \\
\text { Médicos veterinarios } \\
\text { INTA } \\
\text { SENASA } \\
\text { Investigadores } \\
\text { internacionales- } \\
\text { evaluadores revistas }\end{array}$ & $\begin{array}{c}\text { Productor } \\
\text { Laboratorios } \\
\text { UBA } \\
\text { INTA Castelar } \\
\text { INTA Bariloche } \\
\text { CNEA } \\
\text { Northeweastern university } \\
\text { Investigadores- } \\
\text { Evaluadores } \\
\text { internacionales } \\
\text { EULASUR } \\
\text { FAN } \\
\text { USPTO }\end{array}$ \\
\hline
\end{tabular}

Para ellos, los atributos por separado no co-construyen funcionamiento del método.

En el diagnóstico de la brucelosis el método del tacto es el más utilizado por los productores ovinos, particularmente por aquellos que se encuentran en la región patagónica -lugar, por otra parte, donde se intenta implementar el método "nano"-. Si bien a los fines de los investigadores y técnicos el tacto presenta una baja confiabilidad, asociada a la posible confusión con otras patologías, para el grueso de los productores es el método dominante, al que se le atribuye la mayor rapidez en la obtención de resultados (es instantáneo), a la vez que se lo considera el más "práctico" de llevar a cabo.

En cambio, las cuestiones asociadas a la determinación por el método inmunológico, muestran una relación "inversa" respecto del método anterior. Los investigadores y técnicos lo consideran el método más confiable, no sólo por estar ligado a un conjunto de pruebas de laboratorio sino, porque se encuentra validado por constantes intercambios provenientes del sector académico que "perfeccionan" esta técnica, los productores le otorgan menor funcionamiento tanto por el tiempo de espera para los resultados -en muchos casos alrededor de 30 días- como así también por la lejanía con laboratorios y centros urbanos donde procesar las muestras.

La condición de "funcionalidad" otorgada a ambos métodos es central, ya que a partir de los atributos asociados al funcionamiento de cada uno por parte de investigadores y productores, en la actualidad ambos coexisten. Más aún, estudios realizados en otras zonas del país (Suarez et al., 2011) destacan que los dos métodos se utilizan en partes "iguales" para el diagnóstico de brucella ovis.

En este contexto, los investigadores involucrados en el desarrollo del equipo de diagnóstico "nano" atribuyen al mismo una funcionalidad "complementaria" a los dos métodos anteriores. Desde el discurso e ideas planteadas, el equipo presentaría resultados confiables que serían obtenidos de forma instantánea y de fácil utilización por parte de los usuarios.

El planteo llevado a cabo por parte de los técnicos del equipo relaciona directamente las características que 
hacen "superior" al mismo a la utilización de nanotecnologías. Esta relación se puede definir como de tipo "determinista tecnológica" 6 en donde las N\&N van a generar nuevos métodos que suplan las falencias de los anteriores.

Los regímenes de conocimiento involucrados en la utilización y funcionamiento de los diferentes métodos difieren ampliamente. El "tacto" es una operación compuesta por dos tipos de saberes, uno genérico asociado al trabajo del productor en el campo y su labor diaria. El productor se encuentra en dialogo permanente con un conjunto de conocimientos disciplinares y provenientes en su mayoría del Médico Veterinario (MV), uno de los grupos de mayor importancia en el marco de la producción ovina. Este diálogo es el principal componente de la alianza entre MV y productores.

El análisis mediante técnicas de inmunología se basa en su totalidad en un régimen de conocimiento de tipo disciplinar, y está asociado a la medicina veterinaria y las técnicas de laboratorio. El funcionamiento de este método se encuentra condicionado a la interpretación de los MV y el trabajo de los laboratoristas.

En cambio, el método nano se plantea como de tipo transdisciplinar. Para su desarrollo convergen saberes provenientes de la medicina veterinaria (básicamente del sector académico), investigadores en $\mathrm{N} \& \mathrm{~N}$, técnicos y extensionistas de INTA y técnicos de laboratorio. A diferencia de los anteriores no involucran, en principio, aportes de MV que trabajan en campo, como así tampoco de los productores ovinos.

Los tipos de conocimientos asociados a cada técnica varían considerablemente, en el caso del tacto se requieren tanto conocimientos tácitos, como adquiridos a través del proceso de aprender-haciendo y también es necesario el aporte de conocimiento experto. Los primeros provienen del trabajo de los productores, de las técnicas adquiridas en su labor y el traspaso intra e inter generacionalmente asociado al manejo del ganado.

Paralelamente, la interacción entre MV y productores se da en el marco de un proceso de aprender-haciendo. Las habilidades necesarias para la detección de la enfermedad -sólo a través de la vista- requieren no sólo del aprendizaje, sino del trabajo a campo para generar un proceso de mejora continua, que permita detectar la patología con mayor certeza. Por último, el diagnostico se complementa con el conocimiento experto que aportan los MV, adquirido mediante la formación universitaria.

Para trabajar mediante técnicas inmunológicas se requiere en su totalidad el aporte del conocimiento experto, tanto de los MV como intérpretes de los

\footnotetext{
6 "Tradicionalmente, cuando las ciencias sociales piensan la relación tecnología-sociedad lo hacen en el marco de abordajes deterministas lineales: o consideran que la tecnología determina el cambio social (determinismo tecnológico), o consideran que la sociedad determina la tecnología (determinismo social). En la práctica estas maneras de entender la cuestión construyen una separación tajante entre problemas sociales y problemas tecnológicos, como si se tratara de asuntos independientes." (Thomas \& Becerra, 2014: 122).
}

resultados provenientes de los análisis de sangre como así también de los técnicos de laboratorio encargados de realizar dichos análisis. Se puede destacar además la labor de investigadores del sector académico y su aporte mediante publicaciones referidas a esta problemática.

La propuesta de quienes implementan el método nano engloba un conjunto de tipologías de conocimiento amplias y diversas, tales como: el conocimiento experto, cuestiones asociadas a procesos de aprenderhaciendo y en relación a estas dinámicas de aprendizaje por interacción (learning by interacting). El conocimiento experto es el proveniente de los aprendizajes disciplinares de cada uno de los participantes, se destacan tanto el trabajo de MV ligados al sector académico, como así también de Físicos que trabajan en el desarrollo del equipo.

Los conocimientos derivados de procesos de aprenderhaciendo surgen a partir del momento en el cual los MV comienzan a trabajar en el desarrollo del dispositivo en conjunto con los físicos, ya que estos últimos no poseen habilidades para el desarrollo de equipamiento para el trabajo a campo y su posterior implementación. En este caso los conocimientos asociados, básicamente a dinámicas de learning by interacting, están compuestos por los procesos de interacción entre investigadores y productores en la resolución de los problemas que surgen al momento de la prueba del prototipo.

En estrecha relación con el punto anterior, se destaca que las capacidades de I+D necesarias para la implementación de cada uno de los métodos difieren significativamente. En el caso del "tacto", la I+D es escasa, y se basa en los aportes que instituciones como SENASA o INTA, mediante sus investigaciones y contacto con los productores y MV del sector productivo, generan a través del tiempo mejoras incrementales asociadas la técnica y los resultados obtenidos.

El trabajo con muestras de sangre, que surge del método inmunológico, requiere capacidades de I+D que podrían ser definidas como "intermedias" respecto de los métodos analizados. Se trata de procesos con complejidad relativa que en ciertas regiones del país es de amplio alcance para los GSR participantes.

Por último, para el caso del método "nano" se requiere de amplias capacidades de I+D y una densa red de relaciones institucionales para poder ser llevado a cabo. En el caso bajo análisis, trabajaron en forma conjunta instituciones como el INTA y la Comisión Nacional de Energía Atómica (CNEA) a nivel nacional y la Northwestern University a nivel internacional. Paralelamente, fueron necesarias diversas fuentes de financiamiento, tanto internas como externas, para poder completar la construcción del prototipo.

En cuanto a las articulaciones institucionales y la asociación al mainstream científico-tecnológico necesario para el funcionamiento de los métodos de diagnóstico solamente el "inmunológico" y el "nano" requieren de las mismas. El primero al basarse en tecnologías maduras posee una estabilidad relativa que hace que no genere grandes articulaciones con instituciones de I+D, y tampoco necesita de grandes articulaciones al mainstream científico-tecnológico. 
En cambio, la construcción de la metodología "nano" requiere de densas articulaciones institucionales de los investigadores de INTA y CNEA, de neto perfil científico-técnico, para el desarrollo del equipo se basaron en la estrecha relación que poseen con el mainstream científico-tecnológico y las best practices internacionales.

Un punto a destacar son las relaciones usuarioproductor asociadas a cada método. Aunque con matices, los tres conciben su funcionamiento en el trabajo con los productores ovinos. El "tacto" es una metodología que apoya gran parte de su funcionamiento en esta relación, ya que el productor es uno de los principales integrantes de esta alternativa tecnológica.

Si bien el método inmunológico, no requiere la participación del productor para su realización, si se vuelve necesaria en la validación y funcionamiento de la misma. $Y$ cabe señalar que gran parte de los productores ovinos significan a la misma como la herramienta de mayor eficiencia en las estrategias utilizadas en cuestiones asociadas a sanidad animal. De forma similar al primero, en la metodología "nano" los usuarios tienen un alto grado de consideración. Sin embargo, a diferencia del anterior la relación entre usuario y productores se da en dos planos: en el diseño del equipo y en el referido a la validación del mismo. Los investigadores desde un primer momento han hecho referencia a que este dispositivo ha sido diseñado tomando en cuenta todas las problemáticas que poseen los usuarios en la detección de esta patología. No obstante, han establecido que la participación del usuario solo es necesaria para la validación a campo.

Respecto de las estrategias de resolución del problema provenientes de cada metodología, cabe señalar que actualmente las dos primeras se encuentran en coexistencia, y si bien varían en gran parte de sus características, proponen una única solución a esta problemática: la de la eliminación del animal infectado.

El método "nano" se asocia a una solución completamente diferente, y es la vacunación del animal infectado. Desde los investigadores se hace referencia a un "paquete tecnológico" conformado por el dispositivo de diagnóstico instantáneo y una vacuna basada en N\&N como solución a la eliminación de la patología en los establecimientos.

Entre el campo y la academia: alianzas sociotécnicas y métodos de diagnóstico

El funcionamiento de cada método se conforma de un conjunto de alianzas socio técnicas relativamente estables. A continuación, se describen y analizan los procesos de estabilidad y funcionamiento alcanzados por cada uno de los métodos.

\section{El tacto y sus alianzas}

El primero de los métodos analizados, se basa en una alianza de alta estabilidad relativa, conformada por un acotado número de actores, instituciones y artefactos. Dicha estabilidad se apoya en la amplia trayectoria socio-técnica que posee este método en las estrategias de resolución de problemas de los productores, mayormente aquellos que se encuentran en zonas geográficamente desfavorecidas (Figura 1).

En la dinámica de funcionamiento asociada a este método, los grupos participantes difieren respecto de su origen y papel asignado. Sin embargo, se destaca la articulación entre el MV y el productor, siendo escaso para el desarrollo de esta metodología los aportes de las instituciones provenientes del sector estatal.

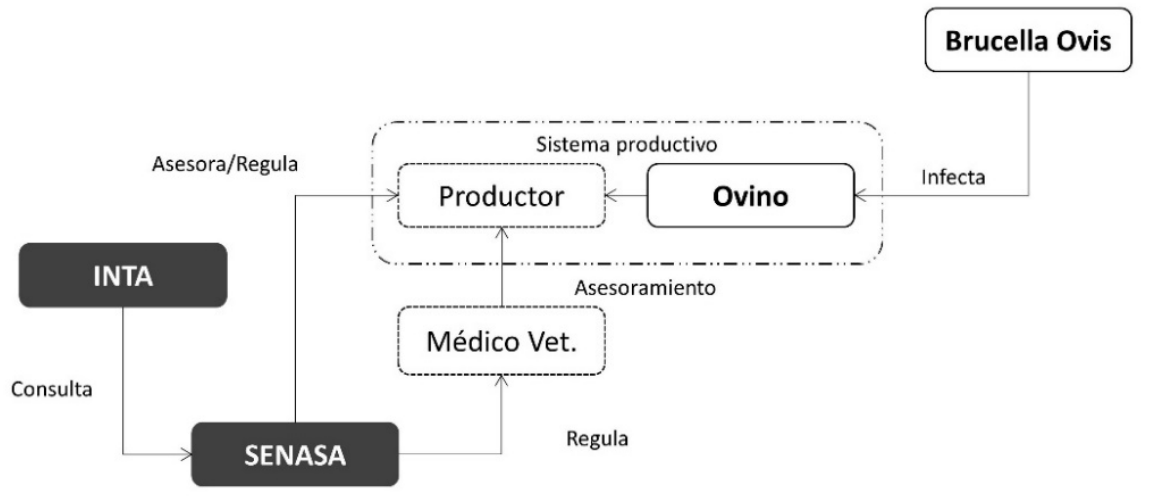

Referencias:

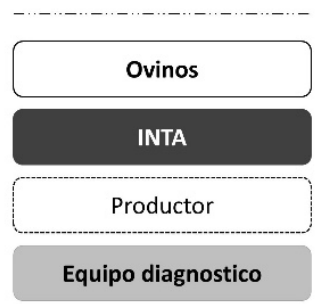

Sectores

Elementos biológicos

GSR sector público

GSR sector privado

Artefacto nano

Figura 1. Alianzas socio-técnicas para el funcionamiento del método basado en el tacto animal. Fuente: Elaboración propia. 
Dos alianzas son las que permiten, casi en su totalidad, el funcionamiento del "tacto". Por un lado, la que se encuentra conformada entre los animales y los productores, basada en el funcionamiento de los sistemas de producción ovina y en la observación de la enfermedad en los animales. Paralelamente, esta alianza se complementa con aquella construida entre el productor y el médico veterinario en el marco de un "dialogo de saberes", tal como se explicó en la tabla 1. La presencia del MV en este método lo convierte en un "punto de pasaje obligatorio" (Callon, 1986), ya que, aunque el productor posea las capacidades para la detección de la enfermedad, es el MV quien lo traduce en soluciones y, a su vez, traduce la información proveniente de los organismos públicos de $I+D$ que tengan algún tipo de influencia sobre el método.

Análisis de sangre e instituciones: alianzas para la construcción del método inmunológico

El funcionamiento del método denominado inmunológico se apoya en un número relativamente importante de alianzas, y -de forma similar al tactoposee una amplia trayectoria socio-técnica en la producción ovina nacional. Se destaca, además el doble significado que posee este método, ya que no sólo es reconocido por productores y MV, sino que a su vez desde el sector académico los investigadores lo utilizan para la realización de ensayos y pruebas a campo. (Figura 2)

La alianza entre productores y ovinos, como así también la conformada por el MV y el productor son centrales para el funcionamiento de este método. No obstante, la sangre se transforma en un componente esencial del esquema de alianzas, ya que se necesita de ésta para poder llevar a cabo la detección de la enfermedad.

El MV posee un papel relevante en la articulación de las alianzas. Sin embargo, en este caso su papel se ve reforzado ya que no sólo es quien "posee" la expertise para detectar la enfermedad y brindar soluciones, sino que también "traduce" el trabajo del laboratorio y los resultados de los análisis de sangre. Paralelamente, los MV provenientes del sector académico necesitan construir una alianza con los MV "asesores" para poder realizar tareas de investigación, ya que son estos quienes brindan información relevante en actividades científicas.

Cuando los átomos y las ovejas ¿Se cruzan? (o las alianzas en torno al diagnóstico "nano")

El diseño e implementación de un equipo basado en N\&N para el diagnóstico de brucelosis "a campo", como se menciona anteriormente, requiere de un conjunto de alianzas socio-técnicas de mayor complejidad y alcance relativo que los analizados previamente. Si bien este método aún se encuentra en la etapa de pruebas de prototipo, en base a los resultados obtenidos hasta el momento es posible identificar las alianzas conformadas para su funcionamiento. (Figura 3)

A diferencia de los anteriores, tanto para el diseño del kit como para su posterior implementación y uso, son los técnicos-investigadores quienes asumen el papel de "punto de pasaje obligatorio", ya que no solo conformaron las alianzas para la construcción del equipo, sino que a su vez son quienes intentan generar las acciones que permitan la incorporación del kit por parte de los productores.

El papel de las instituciones públicas de $1+D$ es sumamente relevante en esta metodología, ya que son quienes proveen tanto los recursos humanos, como materiales y monetarios necesarios para el funcionamiento del equipo. Paralelamente, se destaca el papel del INTA que a través de sus extensionistas busca generar alianzas con los productores para "legitimar" al artefacto.

El origen de este proyecto se remonta a la conformación de un equipo de trabajo por tres investigadores del INTA, los que crearon un grupo de investigación denominado Bionantecnología que forma parte del Instituto de Virología el cual pertenece al Centro de Investigación en Ciencias Veterinarias y Agronómicas ubicados en la dependencia INTA Castelar.

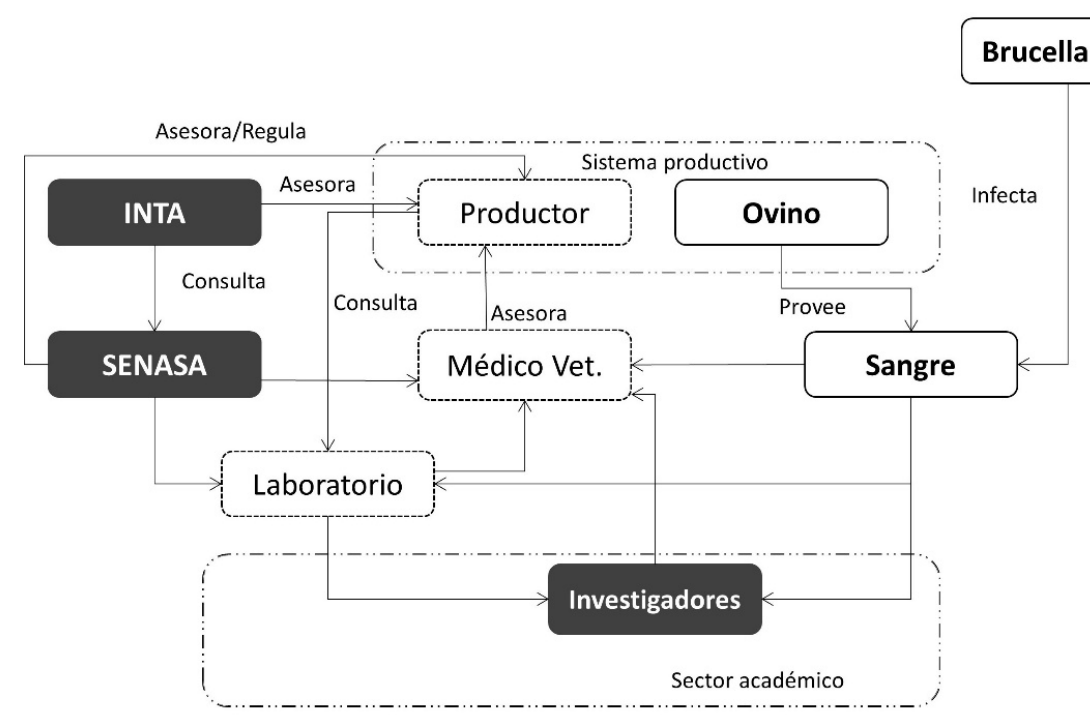

Figura 2. Alianzas socio-técnicas para el desarrollo del método de detección inmunológico. Fuente. Elaboración propia. 


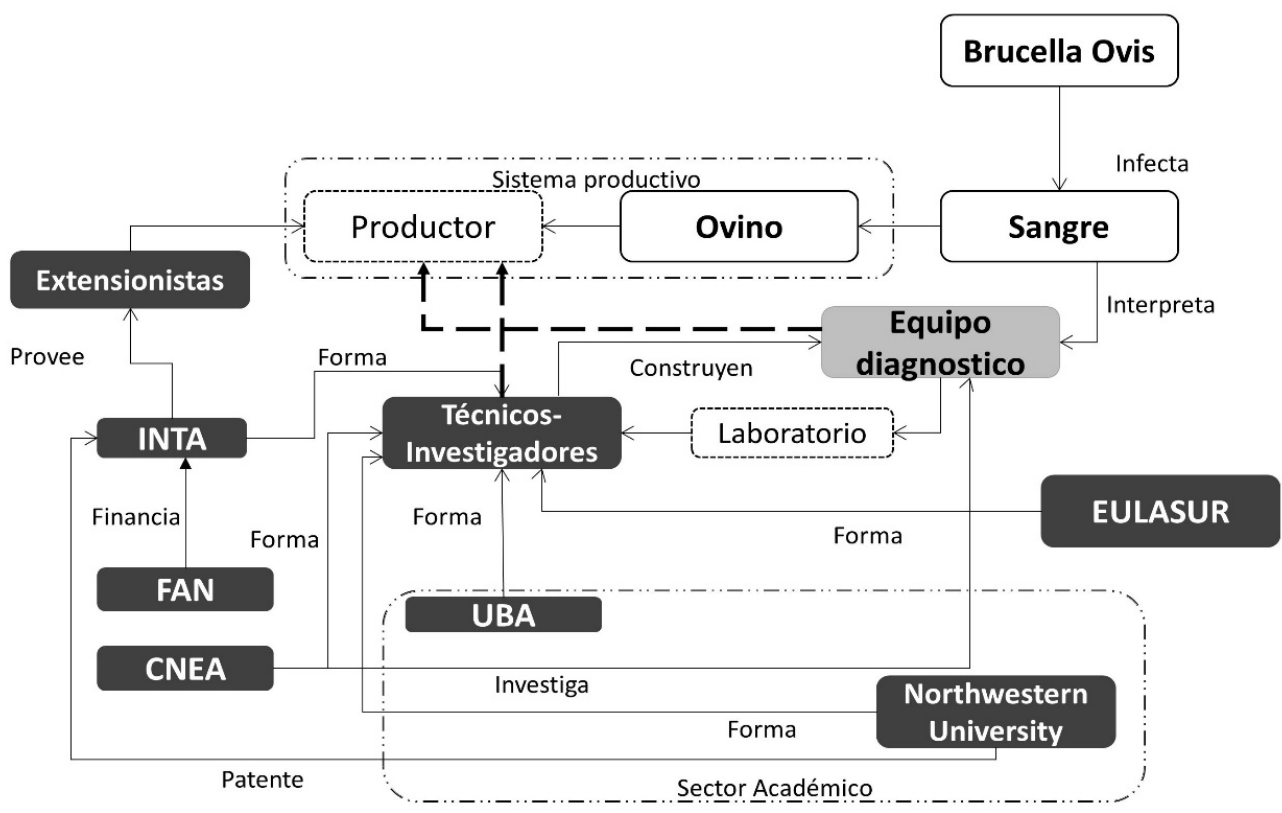

Figura 3. Alianzas socio-técnicas para el desarrollo de un equipo portátil de análisis de brucelosis basado en nanotecnologías. Fuente. Elaboración propia.

Si bien no existió una demanda explicita por parte de la institución en la generación de este grupo, una vez conformado el mismo pasó a formar parte de una estrategia de capitalización en el marco del INTA. La conformación del mismo respondió a varios factores, tanto personales asociados a la búsqueda de inserción institucional por parte de los investigadores, como institucionales, relacionados a la necesidad de visibilidad del INTA dentro del campo de las nanotecnologías. Sin embargo, fue significado por parte de la institución como la respuesta a la búsqueda de soluciones a la problemática.

El sector académico, también posee un papel destacado en el marco de las alianzas socio-técnicas conformadas, ya que gran parte de la información y las "ideas" para el diseño y construcción del artefacto provienen del trabajo de los técnicos-investigadores en el desarrollo de sus investigaciones de posgrado en la Northwestern University.

El sustento de esta metodología parte de una matriz cognitiva completamente diferente a las anteriores, en la que se pueden identificar al menos dos cuestiones: i. el desplazamiento del MV en calidad de asesor para el desarrollo y funcionamiento del método y ii. el papel asumido por el sector académico, que aumenta notablemente la generación del número de alianzas e intermediarios, tanto humanos como no-humanos, requeridos para que el kit pueda ser implementado a campo.

\section{SÍNTESIS Y APRENDIZAJES}

Del análisis en términos de alianzas socio-técnicas existentes en torno a los métodos de diagnóstico de la Brucelosis Ovina se desprenden un conjunto de aprendizajes en diferentes planos de los conocimientos involucrados, las acciones político-institucionales y la planificación estratégica y de desarrollo.

En primer lugar, se destacan las diferencias entre las alianzas que dan sostén y sentido a cada uno de los métodos. Mientras que en los dos primeros es el trabajo conjunto e intercambio entre el MV y el productor el componente principal de la alianza, en el caso del método basado en tecnologías emergentes que pueda conformarse entre el productor y los técnicosinvestigadores provenientes de las instituciones de I+D. En el plano de los conocimientos, en los dos primeros métodos estudiados, se rescata el proceso dialógico entre el conocimiento tácito encarnado en las prácticas de los productores y el conocimiento experto de los veterinarios. En el método denominado "nano" estos conocimientos son desplazados. El conocimiento transdisciplinar que encarna la $\mathrm{N} \& \mathrm{~N}$, el equipamiento requerido y los nuevos problemas cognitivos y de formación de recursos humanos derivados de la aplicación de N\&N plantea nuevos desafíos a las instituciones y entidades públicas y privadas ligadas a la sanidad animal.

En el plano político-institucional se destaca la escasa consideración de un conjunto de actores claves al momento de planificar el proyecto por parte de los investigadores. De este modo, se trabajó solo con instituciones de $1+D$, sin tener en cuenta aquellos organismos con capacidad de regulación o los actores con mayor legitimidad en el territorio.

A su vez, si bien es plausible la preocupación por la inclusión de las tecnologías emergentes a la resolución de problemas productivos en el agro, desde una concepción lineal y determinista tecnológica se genera una tensión entre la capacidad del productor o proveedor de tecnología y usuario, al conceptualizar en forma errónea a este último, al que sólo se lo convoca en la etapa final de prueba del prototipo. 
En el plano de la planificación estratégica y las dinámicas de acumulación, de forma similar a otras experiencias que involucran las tecnologías emergentes, se advierte que los esfuerzos están puestos en el diseño y formulación del prototipo y desarrollo de la innovación, en este caso el kit, no previendo las implicancias o necesidades para su aplicación en etapa posteriores. Por ejemplo, no se plantean articulaciones con las instituciones encargadas de las políticas de control y regulación de la salud animal como el SENASA, tampoco se planifica el desarrollo e incorporación de la innovación en el mediano y largo plazo, ni se considera o se plantean posibles alianzas y articulaciones necesarias con la industria de insumos que proveen las vacunas o los kits. En este sentido, cabe preguntarse ¿Quién fabrica los kits? ¿Quién la vacuna? ¿Quiénes la incorporan? ¿Qué papel juegan los derechos de propiedad intelectual?, entre otros interrogantes.

\section{BIBLIOGRAFÍA}

Aguero, D., Freire, V., Peralta, M., Vigliocco, M., \& Sandoval, G. 2010. Diagnóstico de la cadena ovina en la Provincia de Córdoba, Argentina. Revista Mexicana de Agronegocios, 26(1345-2016-104268), 161-177.

Agrawal, S. \& P. Rathore. 2014. Nanotechnology pros and cons to agriculture: a review. int. j. curr. microbiol. app. sci 3(3): 43-55.

Ali, M. A., Rehman, I., Iqbal, A., Din, S., Rao, A. Q., Latif, A., ... \& Husnain, T. 2014. Nanotechnology, a new frontier in Agriculture. Adv life sci, 1(3), 129-138.

Arrow, K.1962. The Economic Implications of Learning by Doing. Review of Economic Studies XXIX(80): 155173.

Bernardes, P.C., N.J. de Andrade \& N.D.F.F. Soares. 2014. Nanotechnology in the food industry. nanotecnologia na indústria de alimentos. Biosci. j, 30(6): 1919-1932

Bijker, W. 1995. Of Bicycles, Bakelites, and Bulbs. Toward a Theory of Sociotechnical Change. MIT, Cambridge.

Bijker, W. 2005. “¿Cómo y por qué es importante la tecnología?" Redes 11(21): 19-53.

Bouwmeester, H., P. Brandhoff, H.J. Marvin, S. Weigel \& R.J. Peters. 2014. State of the safety assessment and current use of nanomaterials in food and food production. Trends food sci. tech. 40(2): 200210.

Busch, I. 2008. nanotechnologies, food, and agriculture: next big thing or flash in the pan?. Agriculture and Human values, 25(2), 215-218.

Callon, M. 1986. Some Elements of a Sociology of Translation: Domestication of the Scallops and the Fishermen of St. Brieuc Bay. En: Power, Action, and Belief: A New Sociology of Knowledge?. Law, J.(Ed.), RKP, Londres. pp. 196- 233.

Carrozza, T. \& S. Brieva. 2015. "Nanotecnología y Salud Animal: aprendizajes en torno al desarrollo de una estrategia para prevención y tratamiento para la brucelosis ovina". IX Jornadas de Estudios Agrarios y Agroindustriales Argentinos y Latinomericanos. Buenos Aires, Argentina
Chellaram, C., G. Murugaboopathi, A.A. John, R. Sivakumar, S. Ganesan, S. Krithika \& G. Priya. 2014. significance of nanotechnology in food industry. Apcbee procedia 8: 109-113.

Cortes-Lobos, R. 2013. Nanotechnology research in the us agri-food sectoral system of innovation: toward sustainable development. Doctoral thesis. Georgia Institute of Technology, Atlanta. 250 pp.

Dasgupta, N., Ranjan, S., Mundekkad, D., Ramalingam, C., Shanker, R. \& Kumar, A. 2015. Nanotechnology in agro-food: from field to plate. Food Research International, 69, 381-400.

Delgado, G.C. (2009). Nanotecnología y producción de alimentos: impactos económicos, sociales y ambientales.

Ditta, A., M. Arshad \& M. Ibrahim. 2015. Nanoparticles in sustainable agricultural crop production: applications and perspectives. In: Nanotechnology and plant sciences. Nanoparticles and their impact on plants. Siddiqui, M.H., M.H. Al-Whaibi \& F. Mohammad. Springer international publishing. pp. 55-75.

Duncan, T.V. 2011. Applications of nanotechnology in food packaging and food safety: barrier materials, antimicrobials and sensors. j. colloid interf. sci. 363(1): $1-24$.

FAO/WHO. 2010. Expert meeting on the applications of nanotechnologies in the food and agriculture sectors: potential food safety implications: meeting report. World Health Organization: Geneva Suiza. 129 pp.

Foladori, G. \& N. Invernizzi. 2007. Agriculture and food workers challenge nanotechnologies. Disponible en: http://scitizen.com/nanoscience/agriculture-andfood-workers-challenge-nanotechnologies_a-5-937.html Frewer, L.J., N. Gupta, S. George, A.R.H. Fischer, E.L. Giles \& D. Coles. 2014. Consumer attitudes towards nanotechnologies applied to food production. Trends food sci. tech. 40(2): 211-225.

García, M., T. Forbe \& E. Gonzalez. 2010. Potential applications of nanotechnology in the agro-food sector. Ciênc. tecnol. aliment. 30(3): 573-581.

Giddens, A. 1979. Central Problems in social theory: action, structure and contradiction in social analysis. Universidad de California, Berkeley y Los Angeles, California.

Gusfield, J. 1981. La cultura de los problemas públicos. El mito del conductor alcoholizado versus la sociedad excluyente. Arijón, T.B. \& C. Nijelsohn (Traductoras). 2014. Siglo XXI, Buenos Aires. 352 pp. Handford, C.E., M. Dean, M. Henchion, M. Spence, C.T. Elliott \& K. Campbell. 2014.limplications of nanotechnology for the agri-food industry: opportunities, benefits and risks. Trends in food sci. tech. 40(2): 226241.

Kumari, A. \& S.K. Yadav. 2014. Nanotechnology in agri-food sector. Crit. rev. food sci. nutrition 54(8): 975984.

Licha, I. 1995. Perspectivas de los estudios sociales de la ciencia y la tecnología. Redes 2(4): 129-138.

López, G., S. Peña, E. Genero \& N. Lucero. 2010. Brucelosis Ovina en Argentina. Veterinaria Argentina 27: 269-274.

Lundvall, B-A. 1985. Product innovation and userproducer interaction. Aalborg University Press, Aalborg. Lundvall, B-A. 1988. Innovation as an interactive process: from user producer interaction to the national 
system of innovation. In Dosi, G. et. al. (eds.) (Ed.), Technical Change and Economic theory. pp.61-83.

Lundvall, B-A. (Ed.). 1992. National Systems of Innovation: Towards a Theory of Innovation and Interactive Learning. Pinter, Londres.

Mihindukulasuriya, S.D.F. \& L.T. Lim. 2014. Nanotechnology development in food packaging: a review. Trends food sci. tech. 40(2): 149-167.

Mishra, S., A. Singh, C. Keswani \& H.B. Singh. 2014. Nanotechnology: exploring potential application in agriculture and its opportunities and constraints. Biotech today 4(1): 9-14.

Mukhopadhyay, S.S. 2014. Nanotechnology in agriculture: prospects and constraints. Journal of nanotechnology, sci. applic. 7: 63-71.

Myhr, A.I. \& B.K. Myskja. 2014. Nanotechnology in agriculture. Encyclopedia of Food and Agricultural Ethics, 1417-1423.

Noormans, A.G. 2010. Impacto de la nanotecnología en la producción de alimentos. Lámpsakos 4: 28-35.

Paolicchi, F. 2005. Estudios efectuados sobre Brucellosis ovina. Curso de actualización en Manejo Sanitario Ovino. Instituto Nacional de Tecnología Agropecuaria Balcarce.

Parisi, C., M. Vigani \& E.R. Cerezo. 2014. Proceedings of a workshop on "nanotechnology for the agricultural sector: from research to the field". Joint research centre. Working papers no. jrc89736.

Prasad, R., V. Kumar \& K.S. Prasad. 2014. Nanotechnology in sustainable agriculture: present concerns and future aspects. Afr. j. biotechnol 13(6): 705-713.

Querol Sanchis, J. 2011. Cuestiones clínicas, epidemiológicas y diagnósticas de la brucelosis bovina, ovina y caprina. Sitio Argentino de producción animal. [Extraído de: http://www.produccionanimal.com.ar/sanidad_intoxicaciones_metabolicos/enf ermedades_reproduccion/117-Cuestiones_clinicas.pdf] Resch, S. \& M.C. Farina. 2015. Mapa do conhecimento em nanotecnologia no setor agroalimentar. Rev. Administração Mackenzie 16(3): 51-75.
Robles, Carlos. 2008. Brucelosis en carneros por Brucella Ovis (1st ed.). INTA- EEA Bariloche: Instituto Nacional de Tecnología Agropecuaria.

Rosenberg, N. 1982. Inside the black box: Technology and Economics. Cambridge University Press, New York.

Rossi, M., F. Cubadda, I. Dini, M.I. Terranova, F. Aureli, A. Sorbo \& D. Passeri. 2014. Scientific basis of nanotechnology, implications for the food sector and future trends. Trends food sci. technol. 40(2): 127-148.

Samantarai, S.K. \& A.K.K. Achakzai. 2014 Application of nanotechnology in agriculture and food production: opportunity and challenges. Middle-east j. scient. res. 22(4): 499-501.

Scrinis, G. \& K. Lyons. 2007. The emerging nanocorporate paradigm: nanotechnology and the transformation of nature, food and agri-food systems. International j. socio. food agri. 15(2): 22-44.

Sozer, N. \& J.L. Kokini. 2009. Nanotechnology and its applications in the food sector. Trends biotechnol. 27(2): 82-89.

Suarez, V.H., M. Busetti \& M. Real Ortellado. 2011. Prevalencia de enfermedades y manejo sanitario en los sistemas de producción ovina de lana y carne de La Pampa, Argentina. Veterinaria Argentina XXVIII (284).

Thomas, H. \& L. Becerra. 2014. Sistemas tecnológicos para el desarrollo inclusivo sustentable. Voces para el Fénix 37:120-129.

Thomas, H. 2009. Sistemas Tecnológicos Sociales y Ciudadanía Socio-Técnica. Innovación, Desarrollo, Democracia. En Culturas Científicas y Alternativas Tecnológicas. $1^{\circ}$ Encuentro Internacional. Tula Molina, F. (Ed.), MINCyT, Buenos Aires. pp.65-86

Vijayalakshmi, C., C. Chellaram \& S.I. Kumar. 2014. Trendy usage of nanotechnology in agriculture-a review. International conference on science, engineering and management research. pp 1-4.

Warriner, K., S.M. Reddy, A. Namvar \& S. Neethirajan. 2014. Developments in nanoparticles for use in biosensors to assess food safety and quality. Trends food scie. \& technol. 40(2): 183-199. 